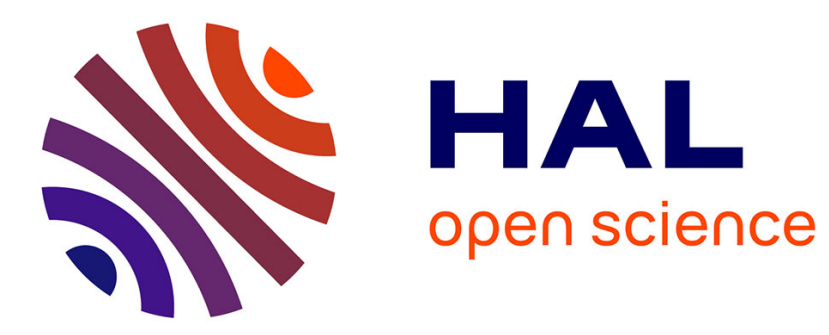

\title{
Evolutionary Games
}

Eitan Altman

\section{- To cite this version:}

Eitan Altman. Evolutionary Games. Tamer Başar. Encyclopedia of Systems and Control, Springer, pp.391-396, 2014, 10.1007/978-1-4471-5102-9_32-1. hal-00927226

\section{HAL Id: hal-00927226 https://hal.inria.fr/hal-00927226}

Submitted on 12 Jan 2014

HAL is a multi-disciplinary open access archive for the deposit and dissemination of scientific research documents, whether they are published or not. The documents may come from teaching and research institutions in France or abroad, or from public or private research centers.
L'archive ouverte pluridisciplinaire HAL, est destinée au dépôt et à la diffusion de documents scientifiques de niveau recherche, publiés ou non, émanant des établissements d'enseignement et de recherche français ou étrangers, des laboratoires publics ou privés. 


\title{
Evolutionary Games
}

\author{
Eitan Altman*
}

\begin{abstract}
Evolutionary games constitute the most recent major mathematical tool for understanding, modelling and predicting evolution in biology and other fields. They complement other well establlished tools such as branching processes and the Lotka-Volterra [6] equations (e.g. for the predator - prey dynamics or for epidemics evolution). Evolutionary Games also brings novel features to game theory. First, it focuses on the dynamics of competition rather than restricting attention to the equilibrium. In particular, it tries to explain how an equilibrium emerges. Second, it brings new definitions of stability, that are more adapted to the context of large populations. Finally, in contrast to standard game theory, players are not assumed to be "rational" or "knowledgeable" as to anticipate the other players' choices. The objective of this article, is to present foundations as well as recent advances in evolutionary games, highlight the novel concepts that they introduce with respect to game theory as formulated by John Nash, and describe through several examples their huge potential as tools for modeling interactions in complex systems.
\end{abstract}

\section{Introduction}

Evolutionary Game Theory is the youngest of several mathematical tools used in describing and modeling evolution. It was preceded by the theory of branching processes [12] and its extensions [1] which have been introduced in order to explain the evolution of familly names in the English population of the second half of the 19th century. This theory makes use of the probabilistic distribution of the number of offspring of an individual in order to predict the probability at which the whole population would become eventually extinct. It describes the evolution of the number of offsprings of a given individual. The LotkaVolterra equations and their extensions are differential equations that describe the population size of each of several species that have a predator - prey type relations. One of the foundations in Evolutionary games (and its extension to population games) which is often used as the starting point in their definition is the replicator dynamics, which similarly to the Lotka-Volterra equations, describe the evolution of the size of various species that interact with each other (or of various behaviors within a given populations). In both the Lotka-Volterra

${ }^{*}$ The author is with INRIA, France. email: eitan.altman@inria.fr 
equations as well as in replicator dynamics, the evolution of the size of one type of population may depend on the sizes of all other populations. Yet, unlike the Lotka-Volterra equations, the object of the modelling is the normalized sizes of populations rather than the size itself. By normalized size of some type we mean the fraction of that type within the whole population. A basic feature in evolutionary games is thus, that the evolution of the fraction of a given type in the population depends on the sizes of other types only through the normalized size rather than through their actual one.

The relative rate of the decrease or increase of the normalized population size of some type in the replicator dynamics is what we call fitness and is to be understood in the Darwinian sense. If some type or some behavior increases more than another one, then it has a larger fitness. the evolution of the fitness as described by the replicator dynamics is a central object of study in evolutionary games.

So far we did not actually consider any game, and just discussed ways of modeling evolution. The relation to game theory is due to the fact that under some conditions, the fitness converges to some fixed limit, which can be identified as an equilibrium of a matrix game in which the utilities of the players are the fitnesses. This limit is then called an ESS - Evolutionary Stable Strategy as defined by Meynard Smith and Price in [7]. It can be computed using elementary tools in matrix games and then used for predicting the (long term) distribution of behaviors within a population. Note that an equililbrium in a matrix game can be obtained only when the players of the matrix game are rational (each one maximizing its expected utility, being aware of the utilities of other players and of the fact that these players maximize their utilities, etc). A central contribution of Evolutionary Games is thus to show that evolution of possibly non-rational populations converges under some conditions to the equilibrium of a game played by rational players. This surprising relationship between the equilibrium of a non-cooperative matrix game and the limit points of the fitness dynamics has been supported by a rich body of experimental results, see [3].

On the importance of the ESS for understanding the evolution of species, Dawkins writes in his book "The Selfish Gene" [4]: "we may come to look back on the invention of the ESS concept as one of the most important advances in evolutionary theory since Darwin." He further specifies: "Maynard Smith's concept of the ESS will enable us, for the first time, to see clearly how a collection of independent selfish entities can come to resemble a single organized whole."

Here we shall follow the non-traditional approach describing evolutionary games: we shall first introduce the replicator dynamics and then introduce the game theoretic intenrpretation related to it.

\section{Replicator dynamics}

In the biological context, the replicator dynamics is a differential equation that describes the way in which the usage of strategies changes in time. They are based on the idea that the average growth rate per individual that uses a given 
strategy is proportional to the excess of fitness of that strategy with respect to the average fitness.

In engineering, the replicator dynamics could be viewed as a rule for updating mixed strategies by individuals. It is a decentralized rule since it only requires knowing the average utility of the population rather than the strategy of each individual.

Replicator dynamics is one of the most studied dynamics in evolutionary game theory. It has been introduced by Taylor and Jonker [10]. The replicator dynamics has been used for describing the evolution of road traffic congestion in which the fitness is determined by the strategies chosen by all drivers [8]. It has also been studied in the context of the association problem in wireless communications [9].

Consider a set of $N$ strategies and let $p_{j}(t)$ be the fraction of the whole population that uses strategy $j$ at time $t$. Let $p(t)$ be the corresponding $N$ dimensional vector. A function $f_{j}$ is associated with the growth rate of strategy $j$ and it is assumed to depend on the fraction of each of the $N$ strategies in the population. There are various forms of replicator dynamics [8] and we describe here the one most commonly usued. It is given by

$$
\dot{p}_{j}(t)=\mu p_{j}(t)\left[f_{j}(p(t))-\sum_{k=1}^{N} p_{k} f_{k}(p(t))\right],
$$

where $\mu$ is some positive constant and the payoff function $f_{k}$ is called the fitness of strategy $k$.

In Evolutionary Games, evolution is assumed to be due to pairwise interactions between players, as will be described in the next section. Therefore $f_{k}$ has the form $f_{k}(p)=\sum_{i=1}^{N} J(k, i) p(i)$ where $J(k, i)$ is the fitness of an individual playing $k$ if it interacts with an individual that plays strategy $i$.

Within quite general settings [13], the above replicator dynamics is known to converge to an ESS (which we introduce in the next section).

\section{Evolutionary Games and ESS}

Consider an infinite population of players. Each individual $i$ plays at times $t_{n}^{i}, n=1,2,3, \ldots$ (assumed to constitute an independent Poisson process with some rate $\lambda$ ) a matrix game against some player $j(n)$ randomly selected within the population. The choice $j(n)$ of the other players at different times is independent. All players have the same finite space of pure strategies (also called actions) $K$. Each time it plays, a player may use a mixed strategy $p$, i.e. a probability measure over the set of pure strategies. We consider $J(k, i)$ (defined in the previous section) to be the payoff for a tagged individual if it uses a strategy $k$ and it interacts with an individual using strategy $i$. With some abuse of notation, one denotes by $J(p, q)$ the expected payoff for a player who uses a mixed strategy $p$ when meeting another individual who adopts the mixed strategy $q$. If we define a payoff matrix $A$ and consider $p$ and $q$ to be column 
vectors, then $J(p, q)=p^{\prime} A q$. The payoff function $J$ is indeed linear in $p$ and $q$. A strategy $q$ is called a Nash equilibrium if

$$
\forall p \in \Delta(K), \quad J(q, q) \geq J(p, q)
$$

where $\Delta(K)$ is the set of probabilities over the set $K$.

Suppose that the whole population uses a strategy $q$ and that a small fraction $\epsilon$ (called "mutations") adopts another strategy $p$. Evolutionary forces are expected to select against $p$ if

$$
J(q, \epsilon p+(1-\epsilon) q)>J(p, \epsilon p+(1-\epsilon) q)
$$

\subsection{Evolutionary Stable Strategies - ESS}

Definition $3.1 q$ is said to be an Evolutionary Stable Strategy (ESS) if for every $p \neq q$ there exists some $\bar{\epsilon}_{y}>0$ such that (3) holds for all $\epsilon \in\left(0, \bar{\epsilon}_{y}\right)$.

The definition of ESS is thus related to a robustness property against deviations by a whole (possibly small) fraction of the population. This is an important difference that distinguishes the equilibrium in populations as seen by biologists and the standard Nash equilibrium often used in economics context, in which robustness is defined against the possible deviation of a single user. Why do we need the stronger type of robustness? Since we deal with large populations, it is likely to be expected that from time to time, some group of individuals may deviate. Thus robustness against deviations by a single user is not sufficient to ensure that deviations will not develop and end up being used by a growing portion of the population.

Often ESS is defined through the following equivalent definition.

Theorem 3.1 [13, Proposition 2.1] or [5, Theorem 6.4.1, page 63] A strategy $q$ is said to be an Evolutionary Stable Strategy if and only if $\forall p \neq q$ one of the following conditions holds:

$$
J(q, q)>J(p, q),
$$

or

$$
J(q, q)=J(p, q) \text { and } J(q, p)>J(p, p) .
$$

In fact, if condition (4) is satisfied, then the fraction of mutations in the population will tend to decrease (as it has a lower fitness, meaning a lower growth rate). Thus the strategy $q$ is then immune to mutations. If it does not but if still the condition (5) holds, then a population using $q$ is "weakly" immune against a mutation using $p$. Indeed, if the mutant's population grows, then we shall frequently have individuals with strategy $q$ competing with mutants. In such cases, the condition $J(q, p)>J(p, p)$ ensures that the growth rate of the original population exceeds that of the mutants. 
A mixed strategy $q$ that satisfies (4) for all $p \neq q$ is called Strict Nash Equilibrium. Recall that a mixed strategy $q$ that satisfies (2) for all $p \neq q$ is a Nash equilibrium. We conclude from the above theorem that being a strict Nash equilibrium implies being an ESS, and being an ESS implies being a Nash equilibrium. Note that whereas a mixed Nash equilibrium is known to exist in a matrix game, an ESS may not exist. However, an ESS is known to exist in evolutionary games where the number of strategies available to each player is 2 [13].

Proposition 3.1 In a symmetric game with two strategies for each player and no pure Nash equilibrium, there exists a unique mixed Nash equilibrium which is an ESS.

\subsection{Example: the Hawk and Dove game}

We briefly describe the Hawk and Dove game [7]. A bird that searches food finds itself competing with another bird over food and has to decide whether to adopt a peaceful behavior (Dove) or an aggressive one (Hawk). The advantage of behaving aggressively is that in an interaction with a peaceful bird, the aggressive one gets access to all the food. This advantage comes at a cost: a Hawk which meets another Hawk ends up fighting with it and thus takes a risk of getting wounded. In contrast, two Doves that meet in a contest over food share it without fighting. The fitnesses are summarized in Table 1, in which the cost for fighting is taken to be some parameter $\delta>1 / 2$.

Table 1: The Hawk-Dove Game

\begin{tabular}{|c|cc|}
\hline & $\mathrm{H}$ & $\mathrm{D}$ \\
\hline $\mathrm{H}$ & $1 / 2-\delta$ & 1 \\
$\mathrm{D}$ & 0 & $1 / 2$ \\
\hline
\end{tabular}

This game has a unique mixed Nash equilibrium (and thus a unique ESS) in which the fraction $p$ of aggressive birds is given by

$$
p=\frac{2}{1.5+\delta}
$$

\section{Extension: Evolutionary Stable Sets}

Assume that there are two mixed strategies $i$ and $j$ that have the same performance against each other, i.e. $J\left(p_{i}, p_{j}\right)=J\left(p_{j}, p_{j}\right)$. Then neither one of them can be an ESS, even if they are quite robust against other strategies. Now assume that when excluding one of them from the set of mixed strategies, the other one is an ESS. This could imply that different combinations of these two ESS's could co-exist and would together be robust to any other mutations. This motivates the following definition of an ESSet [2]: 
Definition 4.1 A set $E$ of symmetric Nash-Equilibria is an evolutionarily stable set (ESSet) if, for all $q \in E$, we have $J(q, p)>J(p, p)$ for all $p \notin E$ and such that $J(p, q)=J(q, q)$.

Properties of ESSet.

(i) For all $p$ and $p^{\prime}$ in an ESSet $E$, we have $J\left(p^{\prime}, p\right)=J(p, p)$.

(ii) If a mixed strategy is an ESS, then the singleton containing that mixed strategy is an ESSet.

(iii) If the ESSet is not a singleton, then there is no ESS.

(iv) If a mixed strategy is in an ESSet, then it is a Nash-Equilibrium (see [13][p.

48 Example 2.7]).

(v) Every ESSet is a disjoint union of Nash Equilibria.

(vi) A perturbation of a mixed strategy which is in the ESSet can move the system to another mixed strategy in the ESSet. In particular, every ESSet is asymptotically stable for the replicator dynamics [2].

\section{Recommended Reading:}

Several books cover evolutionary game theory well. These include $[2,5,8,11$, 13]. In addition, the book The Selfish Gene by Dawkins presents an excellent background on evolution in biology.

\section{Summary and Future Directions}

The article has provided an overview of the foundations of evolutionary games which include the ESS (evolutionary stable strategy) equilibrium concept that is stronger than the standard Nash equilibrium, and the modeling of the dynamics of the competition through the replicator dynamics. It has further provided a brief description of recent developments in this area which include the Markov Decision Evolutionary Games. Evolutionary game framework is a first step in linking game theory to evolutionary processes. The payoff of a player is identified as its fitness, i.e. the rate of reproduction. Further development of this mathematical tool is needed for handling hierarchical fitness, i.e. the cases where the individual that interacts cannot be directly identified with the reproduction as it is part of a larger body. For example, the behavior of a blood cell in the human body when interacting with a virus cannot be modeled as directly related to the fitness of the blood cell but rather to that of the human body. A further developement of the theory of evolutionary games is needed to define meaningful equilibrium notions and relate them to replication in such contexts.

\section{Acknowledgement}

The author has been supported by CONGAS project FP7-ICT-2011-8-317672, see www.congas-project.eu. 


\section{References}

[1] E. Altman, "Semi-linear stochastic difference equations", Discrete Event Dynamic Systems , 19:115-136, 2008.

[2] R. Cressman, 2003, Evolutionary Dynamics and Extensive Form Games, MIT Press, Cambridge, MA.

[3] Daniel Friedman, "Equilibrium in Evolutionary Games: Some Experimental Results" The Economic Journal, pages 1-25, Jan. 1996.

[4] R Dawkins. The Selfish Gene, Oxford University Press, Oxford, UK, (1976)

[5] J. Hofbauer and K. Sigmund, 1998, Evolutionary Games and Population Dynamics, Cambridge University Press.

[6] A.J. Lotka, "Contribution to the Theory of Periodic Reaction", J. Phys. Chem., 14 (3), pp 271-274 (1910)

[7] John Maynard Smith and George R. Price, (1973). "The logic of animal conflict”. Nature 246 (5427): 15-18.

[8] William H. Sandholm, Population Games and Evolutionary Dynamics, MIT Press, 2009.

[9] S. Shakkottai, E. Altman and A. Kumar, Multihoming of Users to Access Points in WLANs: A Population Game Perspective, IEEE Journal on Selected Areas in Communication, Special Issue on Non-Cooperative Behavior in Networking Vol 25 No 6, 1207-1215, Aug 2007.

[10] P. Taylor and L. Jonker, Evolutionary stable strategies and game dynamics. Mathematical Biosciences, 16:76-83, 1978.

[11] Vincent, T. L. and Brown, J. S., Evolutionary Game Theory, Natural Selection $\&$ Darwinian Dynamics, Cambridge University Press, Cambridge England, 2005.

[12] H W Watson and Francis Galton, "On the Probability of the Extinction of Families", Journal of the Anthropological Institute of Great Britain, volume 4, pages 138-144, 1875.

[13] J.W. Weibull, Evolutionary Game Theory, Cambridge, MA: MIT Press, 1995. 